\title{
Enhanced Face Recognition based on PCA and SVM
}

\author{
K. Venkata Narayana \\ Assistant Professor \\ Dhanekula Institute of \\ Engineering \&Technology
}

\author{
V.V.R. Manoj \\ Assistant Professor \\ Dhanekula Institute of \\ Engineering \&Technology
}

\author{
K.Swathi \\ Assistant Professor \\ Dhanekula Institute of \\ Engineering \&Technology
}

\begin{abstract}
Feature Extraction and classification are important aspects of pattern recognition, computer vision. Principal Component Analysis is a well-known feature extraction and data representation technique. But this method is affected by illumination conditions. The combination o PCA an SVM for face recognition is presented in this paper. Before applying Principal Component Analysis preprocessing o images done by using wavelet transform. After PCA is applied or feature extraction. Support Vector Machine is used or classification. Experiments based on using Indian face database. The new technique achieves better performance than using PCA only.
\end{abstract}

\section{Keywords}

Principal component analysis; Preprocessing; face recognition; SVM

\section{INTRODUCTION}

Human identification can be done based on using several features. Face Recognition is one way of human identification. From the past few years the attention of Face recognition is increased in the area of pattern recognition and computer vision. The reason is that face recognition technology can be applied in a wide range of fields, such as identity authentication, access control and so on [1]. Face recognition is different from classical pattern-recognition problems such as character recognition. In classical pattern recognition, there are relatively few classes, and many samples per class. Classification of new data done by interpolating among the training faces data. In case of face recognition, there are many classes, and only a few samples per person, and recognition performed by extrapolating from the training samples. The number of training samples can be only one in numerous applications.

Generally speaking, there are two categories of methods in face recognition [2]. First approach is based on facial features such as eyes, nose and mouth etc. It includes locating of features and then various feature extraction methods can be adopted to construct feature vectors of these facial features. Finally, pattern recognition methods like a KNN can be used to recognize the feature vectors.

The second approach based on the statistical information of the image. It applies statistical method directly over entire training sample images to extract features. Holistic feature extraction of face images is adopted in this approach. PCA representation could be used to implement a very efficient and successful face recognition system. Principal Component Analysis is a statistical method used for reducing the dimensionality of a data set, but at the same time it identifies variations among dataset.

The variations between the images of the same face due to illumination and viewing direction are almost always larger than image variations due to changes in face identity [3]. Two key issues should be solved in order to reduce illumination effect [4]. The first issue is about the representation of data of face image. The second issue is what kind of classifier can be used to classify a new face image.

SVM (Support Vector Machine) is a data classification technique. Applying SVM to computer vision problem has been proposed in recent years. Osuna et al [5] trained a SVM for face detection, where the discrimination is between two classes: face and non-face, each with thousands of examples. For a two-class classification problem, the goal is to separate the two classes by a function which is induced from available examples. It is proved to be good a classifier in pattern recognition. A good classifier is of vital importance in face recognition, so SVM is used in our technique.

\section{PRINCIPAL COMPONENT ANALYSIS (PCA)}

PCA is a statistical dimensionality reduction method and it is widely used technique in the areas of pattern recognition and computer vision for feature extraction and data representation. Kirby and Sirovich [6] applied PCA for representing faces and Turk and Pentland [7] applied PCA for recognizing faces.

PCA is commonly referred to as the use of Eigen faces [7].PCA has become one of the most successful approaches in face recognition in this algorithm each face image may be represented as a weighted sum of he Eigen faces .PCA Eigen faces are particularly suitable to reconstruct the images [8] and is used to extract the Eigen vectors of the face images.

These Eigen vectors can be thought as a set of features which together characterize the variation between the face images[7].Eigen vector can be displayed as a sort of ghostly face which is called as an Eigen face. Let us have a brief view of the principle of PCA [7].

\section{Algorithm:}

Step1: A set of $\mathrm{M}$ images with size $\mathrm{N} * \mathrm{~N}$ can be represented by vectors of size $\mathrm{N}^{2}$

\section{$\Gamma 1, \Gamma 2, \Gamma 3, \ldots, \Gamma M$}

Step2: The average training set is defined by

$$
\psi=\left(\frac{1}{m}\right) \sum_{I=1}^{M} \Gamma \mathrm{i}
$$

Step3: Each face differs from the average by vector

$$
\phi i=\Gamma i-\psi
$$

Step4: A covariance matrix is constructed as follows

$$
C=A A^{T}
$$

Step5: Finding eigenvectors of $N^{2} X N^{2}$ matrix is very difficult. Therefore, we use the matrix $A^{T} A$ of size $\mathrm{M}$ x $\mathrm{M}$ and find eigenvectors of this small matrix.

Step6: If $\mathrm{v}$ is a nonzero vector and $\lambda$ is a number such as $\mathrm{Av}=\lambda \mathrm{v}$ 
then $\mathrm{v}$ is an eigenvector of $\mathrm{A}$ with Eigen value $\lambda$.

Step7: Consider the eigenvectors $v_{i}$ of $A^{T} A$

$$
A^{T} A v_{i}=u_{i} v_{i}
$$

Step8: Multiply both sides by A, we can obtain the result:

$$
A A^{T}\left(A v_{i}\right)=u_{i}\left(A v_{i}\right)
$$

Step9: A face image can be projected into this face space by

$$
\Omega_{k}=U^{T}\left(\Gamma^{k}-\psi\right) ; k=1 \ldots M
$$

\section{SUPPORT VECTOR MACHINES}

\section{(SVMS)}

SVM belongs to kernel methods [9]. Kernel algorithms map data from an original space into a higher dimensional feature space using non-linear mapping [10]. This algorithm is applied to feature space. The high dimensional feature space increases the difficulty for computing the scalar products in the feature space exists. Kernel functions used to compute these scalar products. By using kernel functions there is no need to compute the feature space explicitly.

The SVM method was originally developed as a linear classifier [11]. By utilizing kernel methods it also applied for non-linear mapping of data. The way of data separation by SVM is demonstrated on a simplified example in Fig. 1.

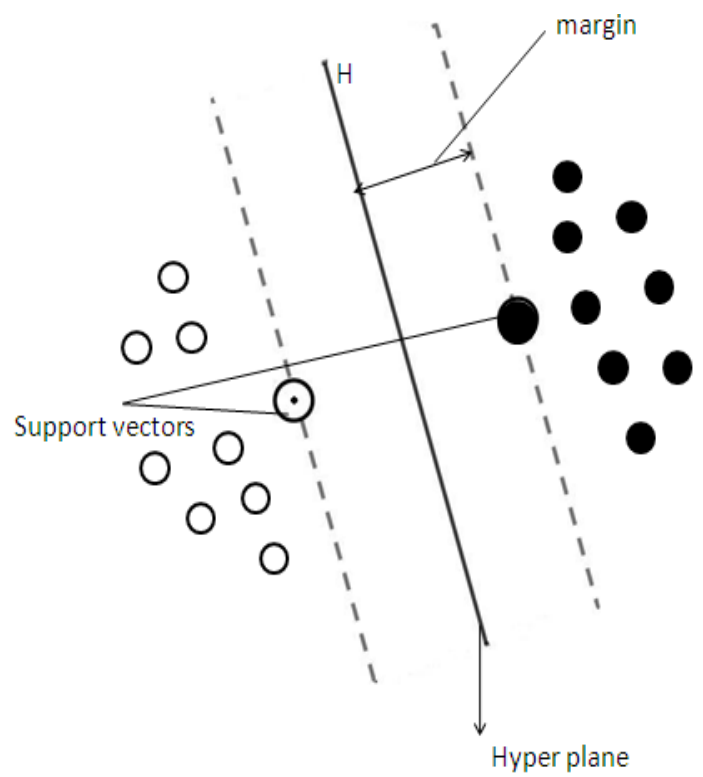

Figure 1:Separation of data using SVM

SVM separates $p$-dimensional data using $p$-1 dimensional decision surface (hyperplane) in such a way that it maximizes the margin of the data sets. The margin is defined as the minimal distance of a sample to the decision surface [9]. The distance of the decision surface (the solid line in Fig. 2) from the nearest appearance of the individual data sets should be as large as possible. In Fig. 2, the dashed lines that are parallel with the hyperplane contain support vectors.

In our tests we use SVM with the RBF (radial basis function) kernel

$$
K\left(X_{i}, X_{j}\right)=\exp \left(-\gamma\left\|X_{I}-X_{j}\right\|^{2}\right), \gamma>0
$$

where $x_{i}, x_{j}$ are data points from the original space. It is important to find optimal parameters $\gamma$ (gamma) and $C$ because different parameter setups are suitable for solving different problems [12]. Using $C>0$ parameter of for determination of a separating hyperplane with the maximal margin in higher dimensional space by SVM..

\section{IMAGE DATABASE}

Tests were performed on a group of 100 grayscale images. image size is $265 \times 195$ pixels. The images differ in head position, lightning conditions, beard, glasses, hairstyle, etc. We selected images of 10 men and 10 women mostly of Caucasian type, but our image set contains also some Asian or African face types. After pre-processing the image size was 81 X81 pixels. Fig 2 shows a sample set of images.
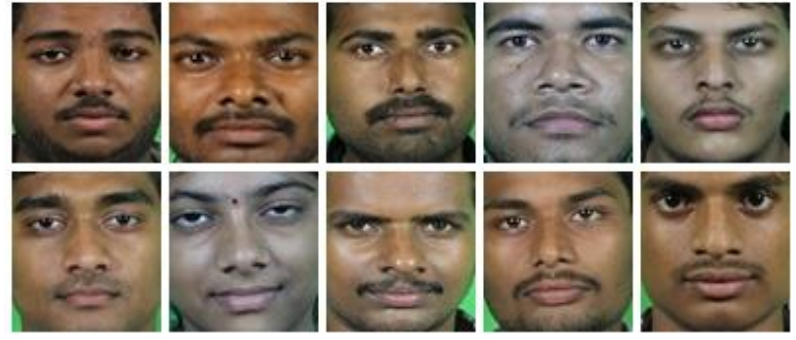

Figure 2: A sample set of images

Pre-processing includes various steps of converting original mage to a normalized image. The normalization Includes convert to gray scale, Geometric normalization, crop the image,Histogram equalization. Fig. 3 shows an example set of Eigen faces.

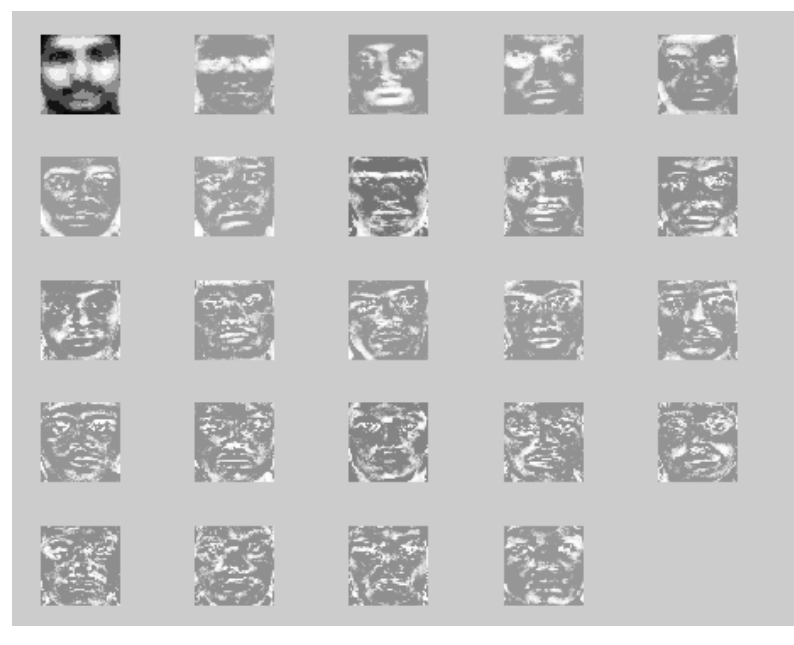

Figure 3: Sample set of Eigen faces

\section{EXPERIMENTS AND RESULTS}

The first step of every pattern recognition system is Feature extraction. The dimension reduction of extracted features is to decrease the time and error of recognition. After preprocessing all images are get in to a single matrix. Each column in the matrix represents a single image. Get the covariance matrix by using this holistic image matrix.. Finally get the feature vectors for the original images. The SVM was trained by using these feature vectors. SVM Classifier classifies a new test image (new data) by applying radial basis function instead of linear classifier.

Before feature extraction the images are to be preprocessed to overcome the lighting conditions, illuminations, head orientations. All the images are to be resized to $81 \mathrm{X} 81$. We consider number of samples of different people. Five image are taken per person. By changing number of samples the recognition rate is also varying. The following table shows the 
recognition rates for different number of samples. Table 1 shows the performance of the system with different samples.

Table 1 A comparison of pca and svm combined with pca in recognition rate $(\%)$

\begin{tabular}{|c|c|c|c|}
\hline $\begin{array}{c}\text { Training } \\
\text { Samples }\end{array}$ & 10 & 20 & 40 \\
\hline PCA & $70 \%$ & $72 \%$ & $80 \%$ \\
\hline PCA+SVM & $89.2 \%$ & $83 \%$ & $86 \%$ \\
\hline
\end{tabular}

\section{CONCLUSIONS}

From the above experimental results we can make a conclusion that the combination of PCA with SVM can give better recognition rate compared with single PCA only. The proposed method notgives higher recognition rate. We will analyze other method in the following papers. In this paper PCA and SVM are combined in face recognition. The recognition rate can be easily influenced by some variations such as lighting, expression and pose in the face images,. Our experiment results conclude that this method can get better recognition rate.

\section{REFERENCES}

R. Chellappa, C.L. Wilson, S. Sirohey, "Human and MachineRecognition of Faces: A Survey," Proceedings of the IEEE, Vol. 83, No.5, May.1995, pp. 705-740.

[1] S. Ranganath and K. Arun, "Face Recognition Using Transform Features and Neural Network," Pattern Recognition, Vol..30,

Oct.1997,pp. 1615-1622.

Adini, Y., Moses, Y., Ullman, S., "Face Recognition: The Problem of Compensating for Changes in Illumination Direction," IEEE Transactions Vol.19, No.7, Jun.1997,pp. 721-732,
[2] G. Guodong, S. Li, and C. Kapluk. "Face recognition by support vector machines," In Proc. IEEE International Conference on Automatic Face and Gesture Recognition, Mar. 2000,pp.196-201.

[3] E. Osuna, R. Freund, and F. Girosi, "Training support vector machines: An application to face detection," Proc. Computer Visionand Pattern Recognition,Vol.3, Jun.1997,pp.130-136.

[4] M. Kirby, L. Sirovich, "Application of Karhunen-Loeve procedure for the characterization of human faces", IEEE Transcvactions on Pattern Analysis and Machine Intelligence vol. 12, pp.103-108, 1990.

[5] M. Turk and A. Pentland, "Eigenfaces for Recognition," J. Cognitive Neuroscience, vol. 3, no. 1, pp. 71-86, 1991.

[6] Yang J, Zhang D, Frangi A.F., and Yang J.Y. "Two dimensional PCA: A new approach to appearance-based face representation and recognition", IEEE PAMI, vol.26, no 1 pp.131-137, 2004.

[7] MULLER, K.|MIKA, S.|RATSCH, G.|TSUDA, K.| SCHOLKOPF, B. : An Introduction to Kernel-Based Learning Algorithms, IEEE Transactions on Neural Networks 12 No.2 (March 2001), 181-201.

[8] ORAVEC, M.|BESZTEDE-S, M.|ROZINAJ, G. : Detection and Recognition of Human Faces and Facial Features, book chapter in ISpeech, Audio, Image and Biomedical Signal Processing Using Neural Networks" (Bhanu Prasad and S. R.Mahadeva Prasanna, eds.), Springer-Verlag, Germany, 2008,pp. 283-306

[9] VAPNIKV. N. : The Nature of Statistical Learning Theory,Springer, 1995

[10] HSU, C. W.|CHANG, C. C.|LIN, C. J. : A Practical Guide to Support Vector Classification, 2008http://www.csie.ntu.edu.tw/ cjlin/papers/guide/guid e.pdf. 